\title{
Serial position functions for letter identification at brief and extended exposure durations
}

\author{
W. K. ESTES, D. H. ALLMEYER, and S. M. REDER \\ The Rockefeller University, New York, New York 10021
}

\begin{abstract}
The properties of serial position functions for tachistoscopic report were investigated over a wide range of viewing times. Four-letter strings of random consonants were presented in varying display locations relative to the fixation point with the observers' eye movements monitored to limit them to a single fixation for each display. Salient properties of the serial position curves include an overall central-peripheral gradient, higher performance at the ends than the interior of letter strings regardless of absolute location, and left-right asymmetry in the visual field, all of these being largely independent of viewing time. Errors reflecting loss of positional information are prominent even at extended viewing times, are more nearly symmetrical in the left and right visual fields than other types of errors, and, in contrast to item errors, occur less frequently in letter sequences that have high frequencies in English. Further, transposition errors exhibit a pronounced peripheral-to-central drift, possibly reflecting gradients of positional uncertainty. Such gradients may be implicated in the peripheral-central asymmetry of the lateral interference effects exerted by other letters on a target letter in a nonfoveal location.
\end{abstract}

Most of our information concerning the perception of letters has come from studies utilizing tachistoscopic exposures of the order of 10 to $200 \mathrm{msec}$. It has seemed necessary to restrict observations to these short exposure times in order to preclude eye movements and to permit the measurement and tracing of information input from a single set of retinal representations of the characters in a display. The short duration and sharp onset and offset of these displays are of course atypical of normal viewing and little is known regarding the generality of the findings or the degree to which concepts derived from tachistoscopic studies can be extended to other conditions. One purpose of the present investigation is to provide some basis for generalization and extension by providing controlled comparisons of some properties of letter identification at brief vs. extended exposure durations.

One of the few studies that have been reported on letter identification with extended viewing time under conditions otherwise similar to those of tachistoscopic studies was reported by Townsend, Taylor, and Brown (1971). Their results concerning the form of serial position curves and the effects of spaces adjacent to letters proved generally similar to those reported by Shaw (1969) from a study utilizing brief exposures. But conclusions that can be drawn from the study of Townsend, Taylor, and Brown (1971) and the follow-up investigation by Taylor and Brown (1972) are limited in two respects. First, although the subjects were instructed to maintain a constant fixation during the prolonged viewing times, fixation

This research was supported in part by Grants MH23878 from the National Institute of Mental Health and GB41176 from the National Science Foundation. Reprint requests may be addressed to W. K. Estes, Rockefeller University, New York. New York 10021. was not actually controlled. Second, no comparison of results obtained with short and long viewing times was obtained for any one experimental situation with other factors controlled. We propose in the present investigation to obtain the desired comparative data utilizing the technique developed by Reder (1972) to monitor fixation.

We shall be concerned particularly with the problem of tracing changes in serial position curves as a function of viewing time and with determining effects of viewing time, position in the visual field, and presence of adjacent letters upon the processing of both item and position information. With regard to form of the serial position curve, a few generalizations can be drawn from the rather extensive literature. When the fixation point is at the center of the display, the serial position curve in terms of percentage of letters correctly identified generally takes roughly the form of a W with performance highest at the center and at the ends of the display but with the ends selectively depressed by pattern premasks and the center selectively depressed by pattern postmasks (Hershenson, 1969; Merikle \& Coltheart, 1972; Merikle, Coltheart, \& Lowe, 1971; Winnick \& Bruder, 1968).

With one exception which will be noted below, all of the studies just cited have yielded serial position curves characterized by symmetry in the left and right visual tields. In contrast, another group of studies, including Bryden (1966), Crovitz \& Schiffman (1965) and one condition in the study of Merikle. Coltheart, and Lowe (1971), yield serial position functions with distinct left-right asymmetry, performance tending to be higher at the left than at the right. A distinguishing aspect of the methodology of the two groups of studies is that the latter results were all obtained with the standard full-report procedure in which the subject 
simply views a display and then reports the letters he has seen. usually by writing them down on an answer card in the proper order. In the former group, the study of Hershenson utilized a report procedure but with prememorized displays; all of the others involved partial report procedures, essentially probes of single positions by means of indicators which appeared following the exposure of the display on a given trial.

One obvious hypothesis which comes to mind as an explanation of the difference between the two groups of studies is that with the full report procedure there is a distinctly greater memory load on the subject and it may be that the left-right gradient simply reflects the greater opportunity for retention loss of items falling later in the report order. To the extent that this hypothesis is well founded, we should expect to find left-right asymmetries progressively attenuated as we proceed from short to prolonged viewing times with consequent reduction of memory load. Another implication of the hypothesis is that we should be able to reduce. if not actually reverse, the left-right asymmetry by training subjects to order their reports from right to left.

With regard to the different types of information, previous studies have varied rather unsystematically between the procedure of scoring protocols simply for the occurrence of letters presented in the display (item scoring) and the scoring of items as correct only if the letter presented in the display occurs in the protocol in its correct position. The former method yields information solely concerning the processing of item information. whereas the latter involves a confounding of item and position information.

In this investigation. we plan to compare the usual measure of item information with a statistically independent measure of position information, namely the conditional probability that an item which is reported from a display is placed in the correct position in the response protocol. There are theoretical reasons for expecting position information under some circumstances to be more sensitive to variables having to do with location in the visual field and distance between characters than measures of item information. In the model outlined by Estes (1972), for example, it is assumed that the density of input channels to feature detectors decreases from the center to the periphery of the visual field. As a consequence, even when the input from a character in a peripheral location successfully activates the appropriate feature detectors and leads to recognition. there will be greater uncertainty concerning the position than would be the case at a more central location, where the distance between input channels is smaller on the average.

In analyzing the data of full reports of tachistoscopically displayed letter strings, one can distinguish not only item and position information but also order information. Independently of the subject's ability to place letters in their correct positions in the display, we may inquire as to his ability to report two letters in their correct left-right order regardless of the positions. Inversions of order do occur in tachistoscopic report data, but they seem not to have been systematically analyzed, perhaps on the assumption that they must simply result from imprecision of memory as distinguished from perceptual processes.

On the basis of the interactive channels model, however, there is reason to expect that inversions of order will occur as a result of perceptual factors and that frequency of these inversions will increase with distance of the displayed letters from the fixation point. It may be possible to obtain some information concerning the extent to which inversions of order result from perceptual as distinguished from memory factors by comparisons of data obtained with short vs. prolonged viewing times. Any effects attributable to limitations of memory should be markedly reduced at longer exposure durations. Thus, if frequency of inversions should prove to be relatively independent of viewing time, we would have support for the assumption of a perceptual interaction.

\section{METHOD}

\section{Subjects}

Three adult female University employees served as subjects and were paid by the session for their services. All three subjects had participated in previous letter-recall experiments using the same display apparatus and had received practice with the eye-movement monitor during the pilot phase of the present experiment. Ail subjects had normal visual acuity and were right-eye dominant.

\section{Apparatus}

The subject sat at a table in a dimly lighted room and viewed the display screen through a $10.7 \times 1.2 \mathrm{~cm}$ aperture in a black vertical reflection shield. On the table directly before the subject was a panel of keys that the subject used to start the display, and just to the right a scoresheet on which the subject wrote her responses at the end of each trial. Vertical bars at each side of the table supported a crossbar, at the center of which a padded headrest was mounted. Use of the headrest prevented gross trial-to-trial variation in either viewing distance or head position in the frontal plane.

Displays were presented on an auxiliary cathode ray oscilloscope connected to a PDP-12 computer which was located in an adjoining control room. The scope rested on the subject's table with the screen approximately $60 \mathrm{~cm}$ from the shield. The scope display area was $23 \mathrm{~cm}$ wide $\times 17 \mathrm{~cm}$ high. Characters displayed on the screen appeared green against a black background and were formed by illumination of the appropriate dots in a 4 by 6 matrix, overall dimensions of which on the screen were $1.00 \times 1.25 \mathrm{~cm}$.

The eye-movement monitor was a modified version of Biometrics, Inc., Model SGH/V.2, operated with filter IN. The subject wore spectacle frames on which the sensor unit of the monitor was mounted. Infrared light reflected from the cornea of the subject's right eye activated a photocell assembly, signals (voltage change) from which were amplified and input to the computer through analog-to-digital conversion channels. Under the conditions of monitor circuitry and programming used in this experiment, only voltage changes produced by lateral eye movements were sampled by the recording system.

\section{Stimulus Materials}

The basic premask array was a horizontal row of 18 identical characters. 9 to either side of the fixation point. a small $x$ displayed at 
the center of the screen. The array subtended a horizontal visual angle of $10.64^{\circ}$ at a viewing distance of $70 \mathrm{~cm}$ and a vertical angle of $0.53^{\circ}$. The central $x$ measured $0.21^{\circ}$ wide and $0.33^{\circ}$ high; the mask characters and letters measured $0.37^{\circ}$ wide and $0.53^{\circ}$ high. Intercharacter spaces were $0.21^{\circ}$. except that the characters adjacent to the $x$ were $0.66^{\circ}$ apart.

The two mask characters utilized will be denoted by the symbols $\$$ and \#, which they resembled, except that in the case of the latter the horizontal bars did not project beyond the vertical lines.

On each trial, the display was produced by replacing four of the premask characters with four uppercase letters drawn at random from a pool of 19 (M. Y, and vowels were excluded). These letters appeared in one of four quadrants: (1) Locations 2.5, (2) Locations 5-8. (3) Locations 11-14, and (4) Locations 14-17. The left- and rightmost locations of each visual field never contained letters. A display with $\$$ mask characters and with letters in Quadrant 1 would take the form:

\section{\$CFQX\$\$\$ $\$$ \$\$\$\$\$\$\$\$}

Quadrant varied randomly from trial to trial. constrained by the use of each quadrant 18 times per 72-trial block. Ten 72-trial display sequences were prepared and used repeatedly throughout the experiment, the sequences for each session being randomly selected.

\section{Design}

The principal experimental variables were exposure duration (150 vs. $2.400 \mathrm{msec}$ ) and mask condition (change vs. no change at display onset). The mask condition will be denoted \$\$, \$\#, or \#\#, the first symbol in a pair indicating the character appearing in the premask and postmask at all locations and the second indicating the character appearing at nonletter locations during exposure of the letter display. At the start of the experiment, subjects were instructed to report the letters they had seen in a left-right (L-R) order, but after the early results became available, revealing unexpectedly pronounced $L-R$ asymmetries in the serial position curves even at the 2.400-msec exposure, an R-L report condition was added.

Each subject contributed a minimum of four 72-trial blocks to each cell of the design, as shown in Table 1. Additional blocks were run whenever the experimenter felt any doubt concerning stability of the data for the given condition.

All six cells of the L-R order were completed prior to reversing order of report. Within each order of report, sessions with the various combinations of mask condition and exposure duration were run in random order, except that in the LR \$\$ condition all subjects did two blocks at 150 msec first.

\section{Procedure}

A 2.400 -msec session began with the subject getting into the spectacle frame and the experimenter positioning the right-eye sensors. In order to determine the monitor tolerance for the session. two xs were displayed at the center of the screen $0.80 \mathrm{~cm}$ apart $\left(0.66^{\circ}\right)$. The subject fixated the left $x$, then shifted her gaze to the right $x$, yielding a voltage change corresponding to an eye movement of $0.80 \mathrm{~cm}$ on the screen. Several of these measures were taken, and halt their median was set as the tolerance.

The subject began each trial with a keypress, which triggered the premask display. When the subject had achieved a stable fixation on the center $x$, she pressed another key to initiate the display of four letters accompanied by the 14 mask characters, at the same time transmitting a voltage signal corresponding to initial eye position. In the \$\# condition, the contents of all display positions changed; in the $\$ \$$ and \#\# conditions. no change was observable in the nonletter positions when the premask characters in the four display positions changed to letters. The letters remained on the screen $2,400 \mathrm{msec}$ or until a monitor voltage was sampled that deviated from the initial voltage by more than the tolerance, in which case the display terminated at once. Voltages were sampled every $5 \mathrm{msec}$.

Display termination was always followed immediately by display of the postmask array (identical to the premask) for $500 \mathrm{msec}$, after which the screen went blank. The subject then wrote her responses on
Table 1

Experimental Design: Cell Entries Indicate Numbers of Blocks Contributed by Each Subject

\begin{tabular}{ccccc} 
& & \multicolumn{3}{c}{ Order of Report } \\
\cline { 3 - 4 } Duration & $\$ \$$ & $\$-R$ & R-L \\
\hline 150 & $6,4,4$ & $4,4,4$ & $4,4,4$ & $5,4,4$ \\
2400 & $5,6,4$ & $6,4,4$ & $5,4,4$ & $4,4,4$ \\
\hline
\end{tabular}

the ruled response sheet. Standing instructions were to write down in the prescribed order the letters that had been identified and to position them just as they had been displayed. Guessing on the basis of partial information was encouraged, but the subject was to leave a position blank if she had no idea what letter had been there. The subjects were told that recall on trials terminated by eye movements was just as important as on trials lasting the full $2,400 \mathrm{msec}$. When the subject had completed her report, she pressed a key to display the premask for the next trial. At the same time, auditory feedback about the duration of the last display was provided via the Teletype in the next room. If the subject had maintained the display for $2.400 \mathrm{msec}$, a short noise was typed out; on monitor-terminated trials, a longer noise was typed. Throughout the experiment, subjects received no other feedback about their responses.

During the course of the experiment, it became evident to the subjects that displays were often terminated even when their gaze had not strayed from the $\mathbf{x}$. Acknowledged eye movements occurred on $10 \%-15 \%$ of the trials, whereas $30 \%-50 \%$ of the trials were terminated by the monitor. The probable cause of these false alarms was the counterrolling of the eyes that accompanied small head movements, without any change in fixation. Rather than introduce the technical changes required to eliminate these false alarms in the midst of the experiment. the experimenter simply informed each subject of the problem and asked her to maintain a steady head position as well as a steady gaze and to disregard the monitor's apparent oversensitivity.

At the end of a 72-trial session, the subject rested briefly, then repeated the tolerance determination as a check against changes in monitor sensitivity due to spectacle movement or weakened state of charge of the monitor.

Sessions at $150 \mathrm{msec}$ used the same procedure, except that eye movements were not monitored. The subject was instructed to fixate the $x$ before pressing the start button. Two 72-trial blocks, with a short rest between, were run in each session.

\section{Clarity Ratings}

Following the $\mathrm{R} \cdot \mathrm{L}$ order of report condition, each subject ran two sessions on a somewhat different reporting task. Instead of identifying the four stimulus letters, the subject was required to rate the clarity of each of the letters, while they were being displayed, and to write down these ratings as soon as the display ended. Clarity was rated on a $0-5$ scale: $0=$ least clear, $5=$ most clear. If an eye movement ended the display before the subject had completed her ratings, she was to give no ratings at ail for that trial. Maximum display duration was $5.115 \mathrm{sec}$, but the subject was to maintain the display for only as long as it took her to rate the four letters, at which time she terminated the display by looking down at the response card.

Two stimulus tapes were drawn at random from the set of 10 , and each subject ran one session with each tape, tape order varying randomly for each subject. Apparatus, calibration, and eye-movement monitoring proced ures were identical to those used in the $2.400-\mathrm{msec} \$ \$$ mask condition with the report procedure.

\section{RESULTS}

Although the data for our three subjects were characterized by exceedingly stable individual 


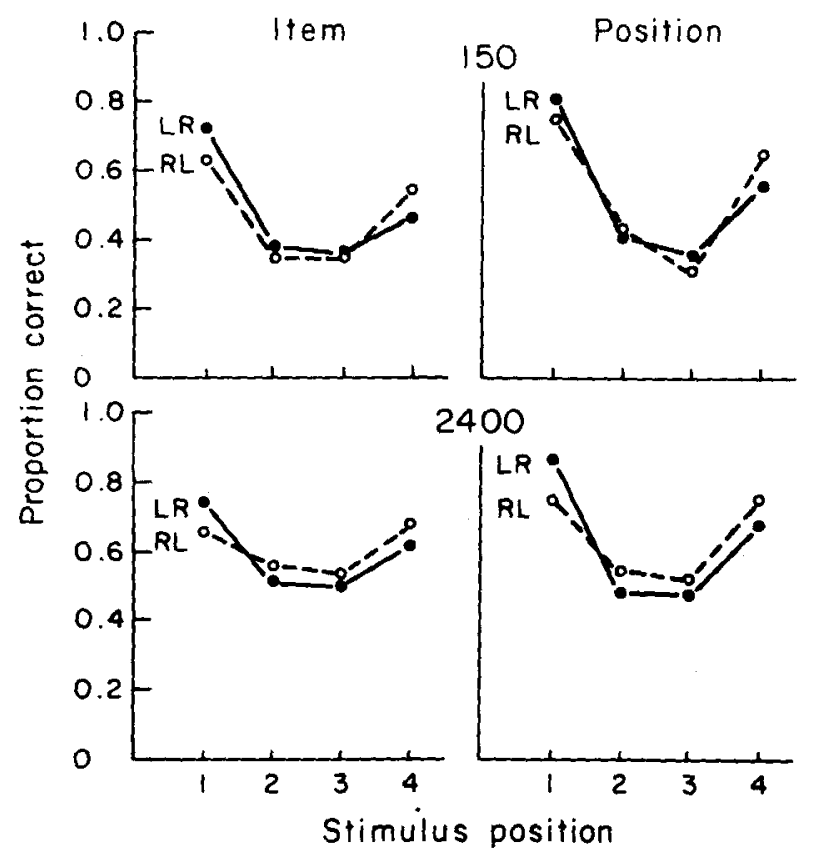

Figure 1. Item and position scores by serial position within a display (dats averaged over quadrants) for left-right (LR) and right-left ( $R L$ ) orders of report at each exposure duration.

differences in detailed forms of serial position functions, all of the main effects and principal interactions involving the independent variables of this study can be brought out without distortion in pooled data. Thus, for all of the analyses to be presented, the data for each subject have been pooled over all sessions run under a particular combination of conditions; the response proportions for individual subjects so obtained are the basic scores which enter into analyses of variance and which are averaged arithmetically to yield the values presented in tables and figures.

\section{Effect of Order of Report \\ Serial Position Curves}

The small, but significant, effects of instructions with regard to order of report may be seen in Figure 1. These data, pooled over quadrants, represent proportions of correct reports, either of items or of positions of correctly reported items, for data obtained with the $\$ \$$ mask condition. For both measures, there is a tendency for the curves representing reports given in the $L-R$ order to be higher at the left and the curves for reports given in R-L order to be higher at the right. The main effect of order is not significant, but for the item data the interaction of Readout Instruction by Duration by Serial Position yields an $F(3,6)=12.7, p<.01$, and for the position measure the interaction of Readout Instruction by Serial Position yields an $F(3,6)=5.7$, $p<.05$. If only the $150-\mathrm{msec}$ condition had been run, one might be tempted to interpret these effects of instructed report order in terms of a memory factor. It would seem quite possible that with respect to retention, items reported early would have an advantage and those reported late might more likely suffer retention loss. However, this interpretation becomes rather implausible when we see that the interactions do not disappear at the $2,400-\mathrm{msec}$ exposure duration where the subject has ample time to rehearse items while the display is still in view and the memory factor should be greatly attenuated.

\section{Effect of Location in the Visual Field}

The overall effect of distance of a letter from the fixation point is a decrease in reportability from center to periphery. as may be seen in the two upper panels of Figure 2, representing proportions of items correct and positions of correctly reported items for the $\$ \$$ mask condition pooled over the $L-R$ and $R-L$. report orders.

Analyses of variance indicate that the trends apparent to the eye in these panels are reliable. For the item measure, the main effects of duration. quadrant, and serial position are all significant at the .01 level $[F(1,2)=139.1, F(3,6)=29.2$, and $F(3,6)$ $=10.4$, respectively], and the interaction of Quadrant by Serial Position is significant at the .05 level $[F(9,18)=3.5]$. For the position measure, the main effect of quadrant yields $F(3,6)=7.3, p<.05$; the main effect of serial position, $F(3,6)=21.6$,

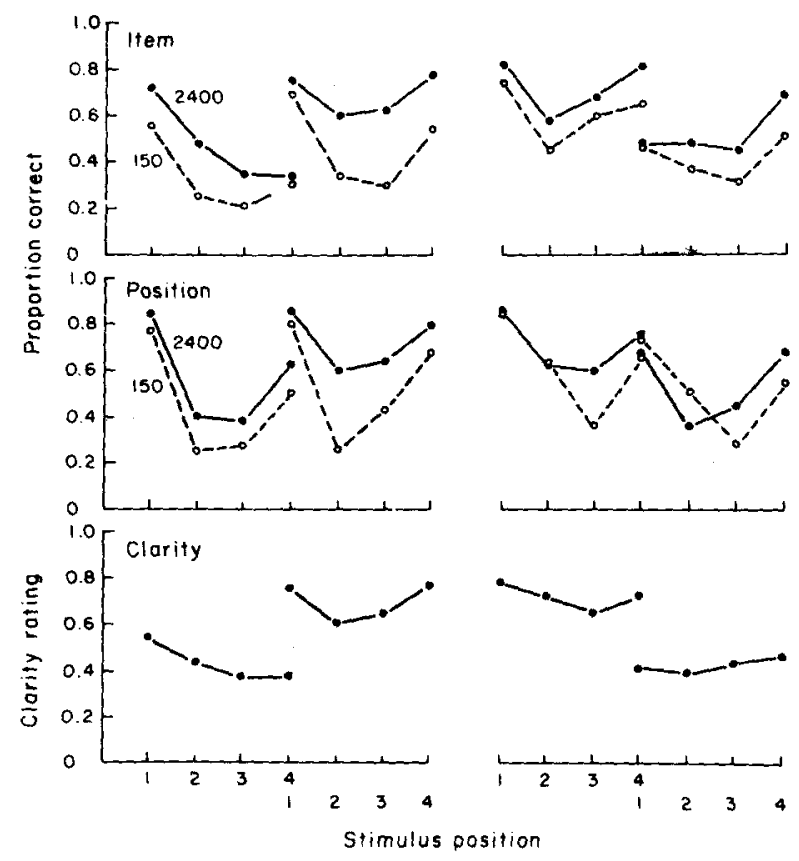

Figure 2. Serial position curves for item, position, and clarity measures, showing the discontinuity effect at the positions in the left and right visual fields where Quadrants 1 and 2 and Quadrants 3 and 4 , respectively, overiap. 
$\mathrm{p}<.01$; and the interaction of Duration by Quadrant, $F(3,6)=14.0, p<.01$.

The central-peripheral gradient is brought out more clearly in Table 2, where various response measures have been averaged over serial positions for each quadrant. In this analysis, and in others to follow, a confusion error is defined as an instance when a stimulus presented in a given position was not reported and its place in the response protocol was taken by another letter, whereas an omission represents an instance when an item was not reported and its place in the response protocol was blank. It may be seen that, for both exposure durations, item information falls off sharply from the central to the peripheral quadrants. The decline from center to periphery is primarily a consequence of an increase in omission errors, though in addition there is a loss of positional information, evidenced by the increase from Quadrants 2 and 3 to Quadrants 1 and 4 in relative proportions of reported items which occur out of position. Positional errors as a percentage of opportunities increase from an average of $36 \%$ to $46 \%$ from the central to the peripheral quadrants at $150 \mathrm{msec}$ and from $27 \%$ to $42 \%$ at $2,400 \mathrm{msec}$.

It is apparent from Table 2 that exposure duration has little or no effect on the probability of reporting items out of position or on the probability of confusion errors, but exerts large effects on the probability of reporting an item in position and on the frequency of omission errors. Since any variation in subjects' thresholds or criteria for guessing would be expected to affect all of these measures, the simplest interpretation seems to be that increasing exposure duration allows the extraction of an increasing amount of information from input characters at all display locations.

The question arises whether the loss of item information from center to periphery simply represents a gradient in visual acuity. The answer is clearly negative if we take acuity in the sense of the individual's ability to resolve the constituent parts of letters. When we presented single letters at the various positions that had been occupied by letters of the four-letter displays in the main experiment (with the same pre- and postmasks but no flanking mask characters during the display), we obtained mean item scores of $99 \%$ correct in central and $96 \%$ in peripheral quadrants at the $150-\mathrm{msec}$ exposure duration. When $\$$ masks were added in the same positions as in corresponding four-letter displays, accuracy in the central quadrants was unchanged, but the mean for peripheral quadrants dropped to $87 \%$. At $2,400 \mathrm{msec}$, the latter condition yielded $99 \%$ correct reports in the central and $97 \%$ in the peripheral quadrants, effectively replicating Townsend. Taylor, and Brown (1971).

Thus nearly all of the decline from center to periphery in reportability of a letter in a given location
Table 2

Effects of Exposure Duration and Quadrant: Percentage Response Breakdown for Data Averaged Over Stimulus Positions

\begin{tabular}{|c|c|c|c|c|c|}
\hline \multirow{2}{*}{$\begin{array}{l}\text { Type of } \\
\text { Response }\end{array}$} & \multirow{2}{*}{$\begin{array}{l}\text { Exposure } \\
\text { Duration }\end{array}$} & \multicolumn{4}{|c|}{ Quadrant } \\
\hline & & 1 & 2 & 3 & 4 \\
\hline $\begin{array}{c}\text { Item Reported } \\
\text { In position }\end{array}$ & $\begin{array}{r}150 \\
2400\end{array}$ & $\begin{array}{l}19 \\
29\end{array}$ & $\begin{array}{l}30 \\
53\end{array}$ & $\begin{array}{l}40 \\
51\end{array}$ & $\begin{array}{l}23 \\
29\end{array}$ \\
\hline $\begin{array}{l}\text { Out of } \\
\text { position }\end{array}$ & $\begin{array}{r}150 \\
2400\end{array}$ & $\begin{array}{l}15 \\
19\end{array}$ & $\begin{array}{l}18 \\
16\end{array}$ & $\begin{array}{l}22 \\
22\end{array}$ & $\begin{array}{l}19 \\
24\end{array}$ \\
\hline $\begin{array}{c}\text { Item Not Rep } \\
\text { Confusion }\end{array}$ & $\begin{array}{r}150 \\
2400\end{array}$ & $\begin{array}{l}30 \\
32\end{array}$ & $\begin{array}{l}30 \\
22\end{array}$ & $\begin{array}{l}26 \\
22\end{array}$ & $\begin{array}{l}30 \\
30\end{array}$ \\
\hline Omission & $\begin{array}{r}150 \\
2400\end{array}$ & $\begin{array}{l}35 \\
20\end{array}$ & $\begin{array}{r}22 \\
9\end{array}$ & $\begin{array}{r}13 \\
6\end{array}$ & $\begin{array}{l}28 \\
18\end{array}$ \\
\hline
\end{tabular}

must be attributed to its interaction with other letters or mask characters simultaneously presented. The differential effects of letters vs. mask characters in this regard will be brought out in the next section.

\section{Effect of Relative Position Within the Display}

At both exposure durations, two main effects of relative position are observed, both superposed on the overall central-peripheral gradient: (1) a decrement in item information, and perhaps even more strongly in position information, in the interior positions of four-letter displays; (2) a central-peripheral asymmetry in the interfering effects of other letters. reflected more strongly in item than in position information.

These effects are manifest in two principal ways. First, in the two peripheral quadrants, one may observe a distinct central-to-peripheral increase in correct response proportions (upper panel of Figure 2) opposed to the overall central-to-peripheral decrease. Second, in both the item and position curves of Figure 2, though more conspicuously in the former, one may observe what we have termed the discontinuity effect. By this term, we refer to the fact that, although the letter in Position 4 of Quadrant 1 is in the same absolute position in the visual field as the letter in Position 1 of Quadrant 2, and the fourth letter of Quadrant 3 in the same position as the first letter of Quadrant 4, one may observe very large differences in reportability of letters at these boundary positions. In other words, we find that a letter flanked peripherally by mask characters and centrally by other letters is much better reported than a letter flanked peripherally by other letters and centrally by mask characters.

\section{Clarity Ratings}

To obtain some additional information as to whether the central-peripheral gradient and the discontinuity effect reflect processes at the perceptual level or at the level of response readout, the data on clarity ratings were obtained and yield the serial 
position functions shown in the bottom panel of Figure 2 (the mean ratings having been converted to a scale of proportions to be more comparable to the response proportions above). The serial position curves for clarity ratings appear generally similar in form to the item response proportions for the 2.400-msec exposure condition. The curves for the clarity ratings are somewhat shallower, but the discontinuities at the boundaries of Quadrants 1 and 2 and Quadrants 3 and 4 are just as pronounced as in the case of the item report measure. Since we have no reason to doubt that our experienced subjects fully understood their instructions, it rather appears that letters which are flanked by other neighboring letters in a peripheral direction simply appear less clear and less perceptible than letters which are flanked peripherally by mask characters.

An analysis of variance of the clarity ratings shows only the effect of quadrant to be significant $[F(3,6)$ $=8.0, p<.05]$. However, there would seem to be no doubt that the principal trends in form of the serial position curves are reliable. All three subjects show a distinct central-peripheral asymmetry in Quadrants 1 and 4 and all three subjects show concave upward curves for Quadrants 2 and 3, with the end positions higher ihan the interior positions. Individual differences in overall level of clarity ratings are rather larger than those observed for correct response proportions, quite possibly owing to criterion differences.

\section{Effect of Changing Mask Characters at Display Onset}

In attempting to determine the source of the discontinuities in the serial position curves at the positions where Quadrants 1 and 2 and Quadrants 3 and 4 overlap, we must recognize that in the data so far considered two variables are confounded. If we consider, say, the comparison of the rightmost letter in Quadrant 1 with the leftmost letter in Quadrant 2, it is the case that only the former is flanked in the peripheral direction by other letters. But it is also the case that with respect to the former the contents of all positions peripheral to the given letter change at display onset, whereas for the latter the contents of those positions remain unchanged at display onset.

In order to separate the effects of change vs. no change from the effects of letter vs. mask characters, we have pooled the data for the $\$ \$$ and \#\# conditions for the item data obtained under the left-right report instructions and present a comparison of these average serial position curves with those of the change $(\$ \#)$ condition in Figure 3 . The effect of a change in the mask characters in nonletter positions at display onset is a drastic reduction in performance, especially at the outer positions in each quadrant, which are, of course, those adjacent to changing vs. nonchanging noise characters.

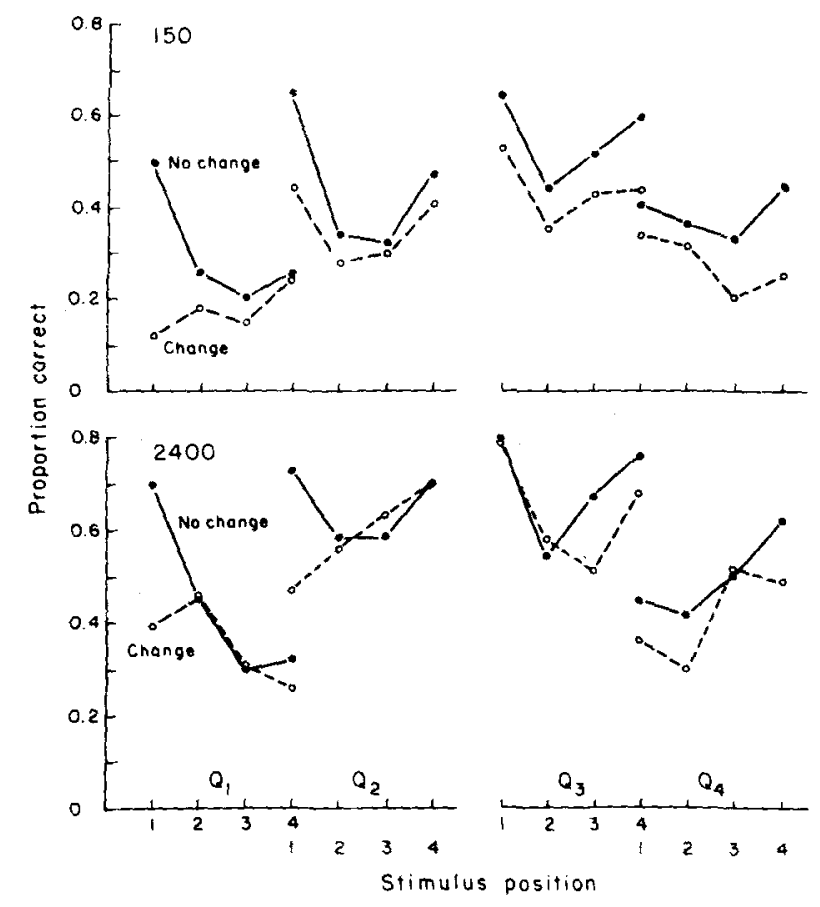

Figure 3. Item curves by display position and quadrant for change and no-change conditions at each exposure duration.

However, the pronounced discontinuities in the item serial position curves at the points of overlap of Quadrants 1 and 2 and Quadrants 3 and 4 are clearly evident even in the change condition. In the analysis of variance for these data. the main effect of mask is significant at the .01 level $[F(2,4)=22.7]$, as are the interactions of Mask by Quadrant $[F(6,12)=5.5]$, Mask by Serial Position $[F(6,12)=6.2]$, and Mask by Quadrant by Serial Position $[F(18,36)=3.5]$. The effects of the change in mask characters on the position measure are generally similar to those on the item measure, but with the smaller Ns the data are not as reliable. For the position data, the effect of change is significant at the .05 level $[F(2,4)=16.9]$ and the interactions of Change by Serial Position $[F(6,12)=$ 12.8] and Change by Duration by Serial Position $[F(6,12)=9.3\}$ are significant at the .01 level, but neither the main effect of quadrant nor the interactions with quadrants approach significance.

Evidently, we must conclude that a letter presented in a given position is masked more by adjacent letters than by adjacent mask characters and more by adjacent noise characters which change at display onset than by adjacent noise characters which do not, and that there is a central-peripheral gradient in the effects of both variables.

The nature of the effect of change in flanking mask characters at display onset can be illuminated further by consideration of specific error categories. To this end, we present in Table 3 a response breakdown analogous to that of Table 2 , but organized by serial 
Table 3

Response Breakdown by Stimulus Position for Change vs. No-Change Conditions With Data Averaged Over Quadrants

\begin{tabular}{|c|c|c|c|c|c|c|c|c|}
\hline \multirow{2}{*}{$\begin{array}{r}\text { Type of } \\
\text { Response }\end{array}$} & \multicolumn{4}{|c|}{$150 \mathrm{msec}$} & \multicolumn{4}{|c|}{$2400 \mathrm{msec}$} \\
\hline & 1 & 2 & 3 & 4 & 1 & 2 & 3 & 4 \\
\hline & \multicolumn{8}{|c|}{ Change Condition } \\
\hline \multicolumn{9}{|l|}{ Item Reported } \\
\hline In position & 10 & 9 & 13 & 11 & 36 & 26 & 21 & 23 \\
\hline Out of position & 27 & 19 & 16 & 23 & 15 & 21 & 28 & 30 \\
\hline \multicolumn{9}{|l|}{ Item Not Reported } \\
\hline Confusion & 19 & 39 & 46 & 21 & 26 & 38 & 37 & 18 \\
\hline \multirow[t]{2}{*}{ Omission } & 45 & 32 & 26 & 45 & 23 & 15 & 14 & 29 \\
\hline & \multicolumn{8}{|c|}{ No-Change Condition } \\
\hline Item Reported & & & & & & & & \\
\hline In position & 44 & 14 & 12 & 26 & 58 & 25 & 25 & 38 \\
\hline Out of position & 11 & 21 & 23 & 19 & 10 & 25 & 24 & 21 \\
\hline \multicolumn{9}{|l|}{ Item Not Reported } \\
\hline Confusion & 22 & 35 & 38 & 26 & 21 & 37 & 35 & 24 \\
\hline Omission & 23 & 29 & 28 & 28 & 11 & 13 & 16 & 18 \\
\hline
\end{tabular}

position for change and no-change conditions at each exposure duration, the data being averaged over quadrants.

It is clear, first of all, that none of the measures show any appreciable effect of the change condition at the interior display positions. A change in the flanking mask characters at display onset affects only report from Positions 1 and 4, that is, the positions adjacent to the changing characters. The nature of the effect is a substantial decrease in items reported, together with an even more drastic decrease in correct localization of reported items, at Positions 1 and 4 at the $150-\mathrm{msec}$ exposure, both effects being still manifest, but reduced in amount, at $2,400 \mathrm{msec}$. Further, the loss of item information reflects sharp increases in omissions at the end positions, the distributions of confusion errors across positions being virtually unaffected by the change variable. Examination of the full stimulus-response matrices reveals that the principal source of the mispositioning of reported letters in the change condition is an increased tendency for letters presented at the end positions of a display to be displaced inward in the response protocol. It is as though subjects were often uncertain as to the precise location of the changing mask character adjacent to the letter display, sometimes interpreting it as having appeared in the adjacent letter position and consequently reporting that letter, if identified, at an incorrect location.

\section{Recruitment of Information During Prolonged Fixations}

Under the 2,400-msec exposure conditions of this study, the display was terminated if an eye movement occurred before the expiration of the full 2,400 -msec interval. On these occasions, the display was immediately replaced by the postmask and the subject proceeded to write down her report just as on other trials. The data for these trials have not been included in any of the other analyses presented, but they do provide an opportunity to examine the course of recruitment of information from the display as a function of exposures of varying durations terminated by eye movements. The EM-terminated trials were grouped according to where the exposure durations fell in the intervals $0-600,600-1,200,1,200-1,800$, or $1,800-2,400 \mathrm{msec}$. Since there proved to be virtually no difference over the last two intervals, those data have been grouped and the resulting curves for short (S), medium (M), and long (L) exposures are presented in Figure 4. The upper panels represent correct response proportions for each of the four quadrants with data pooled over serial positions, and the lower panels proportions as a function of serial position with data pooled over quadrants; the left-hand panels present item and the right-hand panels position scores.

It may be remarked first of all that there is no qualitative difference between exposures terminated by the experimenter and exposures terminated by eye movements. The item curves for the $S$ condition in Figure 4 are so nearly identical in the case of item scores to the corresponding functions for $150-\mathrm{msec}$ exposures that the corresponding pairs of curves could hardly be distinguished by the eye if they had been plotted in the same figure. Nearly the same is true for

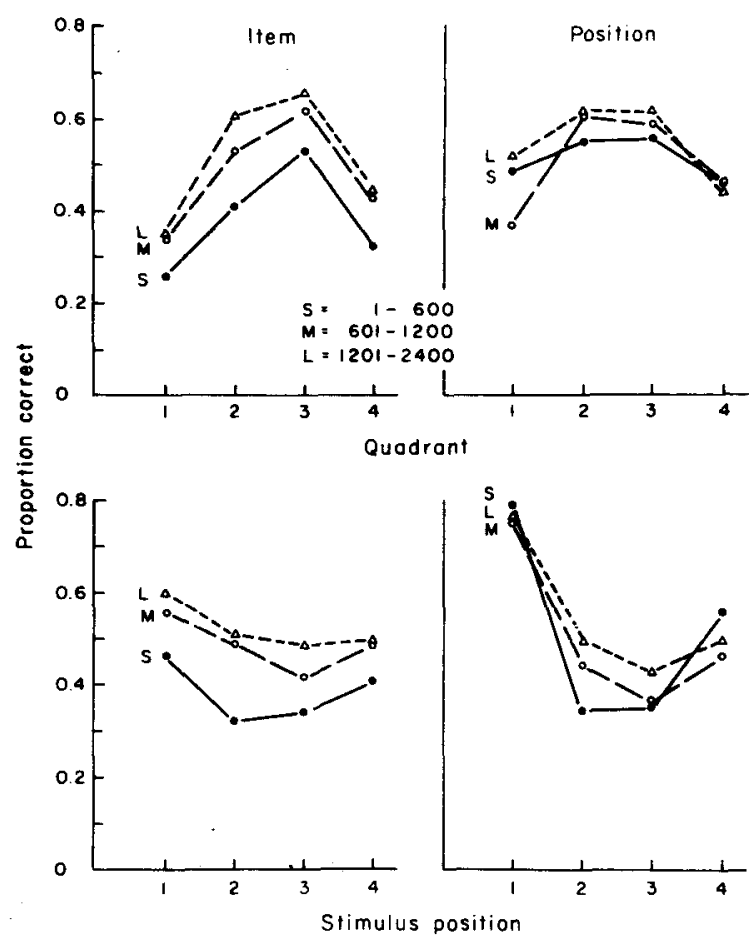

Figure 4. Changes in item and position information by quadrant (upper panels) and by display position (lower panels) for short, medium, and long exposures on trials terminated by eye movements. 
position scores, except that the curves for the $S$ condition run very slightly above those for $150 \mathrm{msec}$. The data presented in Figure 4 represent a pooling over the three mask conditions, but all with the L-R report instruction.

Perhaps the most striking result of this analysis is the contrast between item and position measures. It might have been supposed that position errors observed with brief exposures result from the difficulty in maintaining only partially encoded positional information in short-term memory. But this notion receives no support from the analysis presented in Figure 4. It is the item measures which show a substantial and orderly recruitment with exposure duration. In contrast, for the position measure. variation in proportion of correct responses with exposure is much smaller, less regular, and confined almost entirely to the central quadrants and the interior serial positions. Clearly, positional errors result from factors which operate thoughout the exposure of a display and continue to exert their effects so long as a constant fixation is maintained. It may be noted also that the left-right asymmetries observed with respect to serial positions for both measures and with respect to quadrants for the item measure show no tendency to disappear with increasing exposure duration, suggesting again that these asymmetries, although related to report instruction, are not attributable primarily to limitations of short-term memory.

\section{Error Patterns \\ Directionality of Transposition Errors}

The discontinuities abserved between the serial position curve for the first and second and the third and fourth quidrants suggest that a letter in a given position is nore strongly masked by other letters peripheral than by other letters central to its location in the visual field. The most parsimonious interpretation, of this asymmetry which comes to mind is that it is derivative to a central-peripheral gradient in positional uncertainty. On the basis of our quadrant difterences in position scores, it appears that there is a central-to-peripheral gradient in the individual's ability to localize accurately the position of a character in the visual field. On this assumption, it is more likely that a letter perceived toward the periphery, its position being imperfectly localized, would be assigned by the subject to a more central position than that a character perceived near the fixation point would be assigned incorrectly to a more peripheral location. An immediate implication of this analysis is that we should expect transposition errors on the average to be biased in the direction of movement from the periphery toward the fixation point.

To evaluate this prediction, we have analyzed the data for the $\$ \$$ mask condition. including both L-R and $R-L$ report orders and the $150-$ and $2,400-\mathrm{msec}$ exposure durations. For each combination of these variables, we prepared for each quadrant a stimulus-response matrix giving the frequencies with which letters occurring in each of the four stimulus positions of a display appeared in each of the four serial positions of the subject's response protocol. For each of these tables, two scores were obtained for each subject: (1) the total frequency of leftward transpositions (for example, a letter appearing in Stimulus Position 4 being assigned by the subject to Position 3), and (2) a score representing rightward transpositions (for example, a stimulus appearing in Position 2 of a display being assigned by the subject to Position 4). An analysis of variance of these scores revealed a highly significant interaction of directionality of transpositions with quadrants $[F(3,6)$ $=31.4, p<.001]$, but none of the main effects and none of the other interactions approached significance. The significant interaction of Directionality by Quadrant arises from an increased tendency of leftward transpositions to occur in the two right-hand quadrants and an increased tendency for rightward transpositions in the two left-hand quadrants, thus confirming the expectation that transposition errors would tend to move from the periphery toward the center of the field.

A problem in interpreting this last analysis arises owing to the fact that opportunities for transposition errors depend on the frequency with which letters presented at various positions are reported. Therefore, we have carried out a second analysis, in which the data for Serial Positions 2 and 3 of each quadrant are summarized in a score denoting the percentage of rightward transposition errors relative to the total number of transposition errors. For Quadrants 1 and 2, the mean scores were .62 and .68. and for Quadrants 3 and 4, they were .27 and .32, respectively, in each case signifying an approximately 2:1 excess of transpositions toward the fixation point over those toward the periphery. An analysis of variance of these scores yields a significant main effect of quadrant $[F(3.6)=22.2, p<.01]$, reflecting the central-peripheral trend, but neither the main effect of exposure duration nor the interaction of duration with quadrant approaches significance.

Finally, we may ask to what extent transposition errors may be interpreted in terms of a tendency to fill perceptual gaps. That is, do transpositions predominantly terminate in the positions of letters which were not reported, as distinguished from positions of letters which were reported. from the given display? Denoting the letter presented at position $i$ by $L_{i}$, we shall speak of a "sink" at position $i$ if $L_{i}$ was not reported on the given trial. The analysis required deals with the tendency of a transposition error either to enter a sink or to fill the place of a letter which was reported from the display but out of its 
Table 4

Relative Frequency of Transposition Errors Originating at Interior Locations in Relation to Presence or Absence of a Sink in the Terminal Location

\begin{tabular}{|c|c|c|c|c|c|c|c|c|}
\hline & \multicolumn{8}{|c|}{ Quadrant } \\
\hline & \multicolumn{2}{|c|}{1} & \multicolumn{2}{|c|}{2} & \multicolumn{2}{|c|}{3} & \multicolumn{2}{|c|}{4} \\
\hline & $\mathrm{P}$ & $\mathrm{C}$ & $\mathbf{P}$ & C & C & $\mathbf{P}$ & $\mathrm{C}$ & $\mathbf{P}$ \\
\hline Sink & .47 & .55 & .31 & .69 & .76 & .41 & .57 & .33 \\
\hline Nonsink & .26 & .34 & .20 & .43 & .47 & .24 & .45 & .27 \\
\hline
\end{tabular}

Note. $P$ and $C$ denote transpositions that move peripherally or centrally in the visual field.

correct position. So that the results can $t_{s}$ related to central-peripheral directionality. we have again limited the analysis to letters presented at Positions 2 or 3 of a display. The score computed is based on all cases in which $L_{2}$ or $L_{3}$ was reported from a display but was not placed in its correct position in the response protocol. The values presented in Table 4 represent the proportions of these cases in which the letter appeared in the response protocol at the position (sink or nonsink) one step central (C) or one step peripheral $(P)$ from its correct position.

Several trends are immediately apparent. Firstly. the proportions are uniformly higher for transpositions terminating in sinks than for those terminating in nonsinks, the difference being greater on the average for the central than for peripheral quadrants and for the left as compared to the right visual field. Secondly. the tendency for transpositions to move centrally (i.e.. toward the fixation point) obtains throughout the table. but appears to be essentially independent of whether the error terminates in a sink or nonsink. Finally. the central drift of transpositions is substantially greater in the central than in the peripheral quadrants.

Errors reflecting only order information. In our analyses of transposition errors. we cannot be sure to what extent the observed errors arise from uncertainty associated with the subject's ability to localize individual letters within a display and to what extent they might arise from a tendency to displace all of the letters reported from a display in one direction or the other. In order to obtain some indication of the variables which affect the processing of positional information when errors involving shifts of the entire display are excluded. we have examined errors which involve inversions of order of adjacent letters in the stimulus displays. For this analysis. we consider all instances in which two letters presented in adjacent positions in the stimulus string both appear in the subject's protocol on the trial. and the statistic we shall deal with is the proportion of these instances in which the order of report of the two letters is incorrect. If uncertainty with regard to position of characters arises primarily as a result of fallibility of short-term risual memory, we might expect that these inversion errors would occur with some frequency under the 150 - msec exposure condition but would be very scarce at the prolonged viewing time of $2.400 \mathrm{msec}$. where memory could scarcely be a factor. On this point, our data are decisive: The incidence of inversion errors is surprisingly large, running to about $15 \%$, and proves to be almost entirely independent of exposure duration. The differences between the 150and $2.400-\mathrm{msec}$ exposures are not consistent over other combinations of conditions, and the maín effect of this variable is entirely insignificant.

With one exception, the only variables which significantly affect the frequency of inversion errors are position of the given letter pair within the display and in the visual field. Analysis of variance of the $\$ \$$ data, taking account of both read-out orders and both durations, reveals a significant effect of quadrant $[F(3.6)=6.09 . p<.05]$ and of position within the display $[F(2.4)=14.48, p<.05]$. The proportions of inversion errors giving rise to thse two main effects are $.17 . .13 . .12$, and .20 for Quadrants $1-4$, respectively, and $.11, .23$, and .14 for Positions 1-2. 2-3, and 3-4. respectively. Thus these data agree entirely with the picture exhibited by the serial position curves for positional information, showing the uncertainty with regard to position of perceived characters to increase from the center toward the periphery of the visual field and to be greater in the interior than at the ends of displays at all positions in the field.

The only other significant determiner of inversion errors is change vs. no change in the mask characters in nonletter positions at the time of display onset $[F(1.2)=39.34 . p<.05]$. the direction of the effect being, of course, an increase in inversion errors under the change condition. Although the interaction of change is. no change with serial position did not reach significance. there was a clear tendency for the change condition to increase the frequency of inversion errors at Positions 1-2 and 3-4 while leaving them essentially unchanged for Positions 2-3. For the change condition. inversion frequencies were .22. .26. and .25 for Pairs 1-2, 2-3, and 3-4. respectively. whereas for the no-change condition. they were .11 . .25 . and .18. This result is what would be expected if the increased accuracy of localization of characters at the ends of a display results at least in part from an encoding of auxiliary information concerning the positional relationship between an end character and an adjacent unchanging noise character.

Letter-mask interactions. It is clear that identifiability of a letter in a particular location is reduced in the presence of other letters in a display. even at prolonged viewing times. A major task for research in this area has been that of attempting to determine the level or levels at which the interactions occur that are responsible for this reduced identifiability. One component of the effect has to do with confusability between letters. Thus, in 
experiments with quite different designs, it has been shown that, once the confusabilities of various pairs of letters have been evaluated, one can predict with considerable accuracy the identifiability of a given letter as a function of the number of other letters highly confusable with it which are present in the same display (Estes, 1974). This effect, presumably occurring at the decision level, is related to the separation of letters in a display, but the effect does not disappear at relatively wide separations, and in fact is detectable even when the letters are presented successively rather than simultaneously (Eriksen \& Collins, 1967).

But also there is evidence for interactive effects among characters in a display which appear unrelated to confusability and which apparently must be assigned to the perceptual rather than the decision level (Bjork \& Estes, 1973). Since we have used two different mask characters in the present experiments, it seemed possible that appropriate analysis might reveal differential effects of these characters upon letters which shared specific features with them. In scanning our data for any such effect, we noted what appeared to be a special tendency for a letter containing a vertical line segment as a component to be masked more strongly by the \# than by the \$ mask. In order to examine this possibility systematically, we categorized the letters used in the display into two classes: Letters having a vertical line segment at the left (B. R, etc.) are assigned to what we shall term the $B$ class and the remaining letters to what we shall term the $C$ class. Serial position curves, with item scoring, for letters of the two classes at short and long exposure durations and on trials with $\$ \$$ vs. \#\# masks are presented in Figure 5. An analysis of variance of these data yields borderline significance for the main effects of exposure duration, mask type, and letter group $[F(1.2)=24.82,37.30$, and 21.32 , respectively, $\mathrm{p}<.05$ ) and clearly significant interactions of mask type with letter group, quadrant, and serial position $\{F(1,2)=183.44, F(3,6)=22.98$, and $F(3,6)=$ 12.50 , all having $\mathrm{p}<.01]$.

The expectation of rather specific interactions of masks and letters depending upon particular components seems clearly to be borne out. It may be noted first of all that the interior letters, that is, those in Positions 2 and 3 of a display, are affected very little by the nature of the flanking mask characters. Letters at the end positions of the displays are much more strongly affected, with. in particular, B class letters being much more strongly masked by the \# characters and with this effect most strongly manifest when the B class letter falls at the left end of the display where its vertical line segment would be adjacent to that of a \# mask character. A similar examination of position scores showed a generally similar pattern, except that the differential effects of the two types of masks were much reduced as compared to the effects on the item scores and probably entirely insignificant.

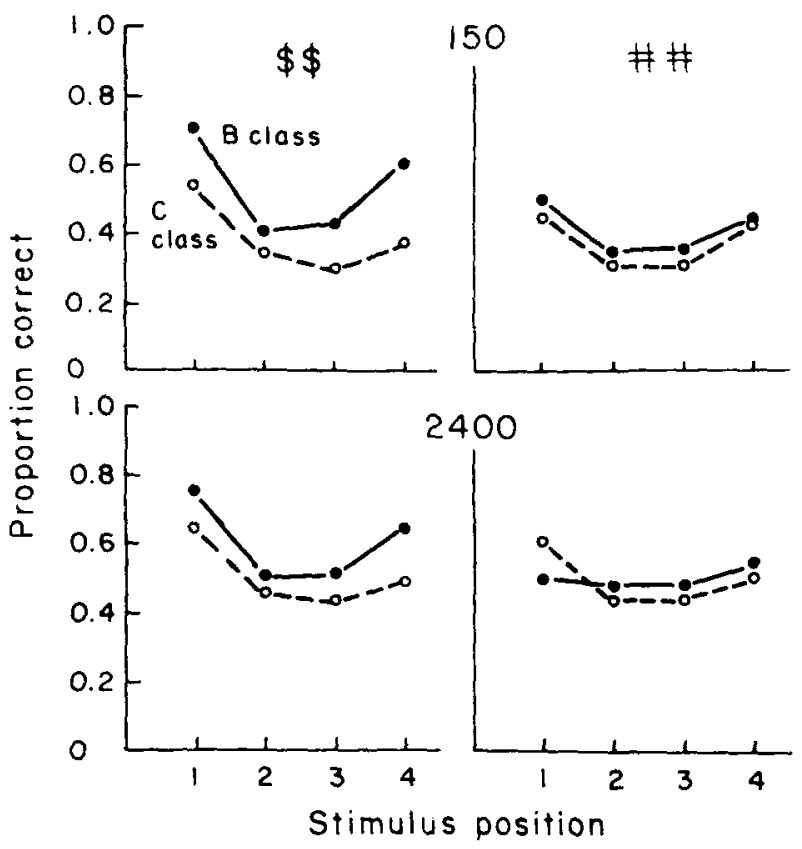

Figure 5. Serial poaition curves averaged over quadrants for data categorized by letter class and by type of mask character.

Effects of familiarity of letter sequences. It is a well-established result that report from tachistoscopically presented letter strings is strongly influenced by the familiarity of the material (see, for example, the review by Neisser, 1967). Under usual experimental conditions, this facilitation is doubtless largely attributable to effects at the level of response bias and rehearsal. As the letter sequences presented increasingly approximate normal English, subjects can utilize redundant information from context to fill in gaps in what they perceive by sophisticated guessing; also. by rehearsing familiar sequences which have once been perceived, they can overcome limitations of short-term memory which otherwise would restrict the amount of material reportable. Under the prolonged viewing condition of the present study, it might be expected that the facilitative effects of familiarity would be greatly attenuated or even eliminated. First, the subjects were well trained and instructed to report what they saw and to avoid sheer guessing; and, second, with the long viewing time, limitations of memory should be a minor factor. Nonetheless, an analysis of this problem by one of the authors (Estes. 1975) suggests that even under these conditions an effect of familiarity should appear. but should be manifest in a selective improvement in the processing of positional as distinguished from item information.

Sone evidence regarding this expectation was obtained from the present study by analyzing the protocols from our pooled data, a total of 105 blocks including 22,680 observations. For each pair of adjacent letters in the display sequences of these 
blocks. a familiarity index was computed by referring to the tables of digram letter frequencies in standard English presented by Underwood and Schulz (1960). The index we utilized was the frequency of occurrence of the given digram divided by the sum of its frequency of occurrence and the frequency of occurrence of the digram comprising the same pair of letters in the reversed order. Digrams such as $\mathrm{CH}$ and $\mathrm{CK}$ receive values of this index near 1.0 , since the letters nearly always occur in these orders and rarely in the reverse order, whereas the digram $\mathrm{RT}$ receives a value near .25 .

Utilizing all of the instances in our data of adjacent letter pairs which were reported with frequencies greater than 10 and for which the corresponding tabled digram frequencies were at least 100 , we first considered frequency of report of the digram (without regard to order of the letters) as a function of the familiarity index. There proved to be virtually no relationship. the frequency of report being .22 for digrams with familiarity indices greater than .5 and .20 for those with indices less than .5 and an entirely insignificant chi square. To bring out the effect of familiarity on the processing of order information, we categorized all of the instances in which letter pairs were correctly reported into those in which the reports were in the correct as distinguished from the incorrect order. A fourfold table of correct or inverted order vs. high- or low-familiarity index yields a chi square of 4.2. $p<.05$. with the relationship being in the expected direction, namely a higher frequency of inverted report orders for digrams with low familiarity ratios.

\section{DISCUSSION}

\section{Summary of Principal Findings}

Viewing time. ${ }^{1}$ Perhaps our most striking finding is that, when normal eye movements are precluded by monitoring the subject's maintenance of fixation, very large variations in viewing time yield no qualitative changes in patterns of performance with respect to various independent variables. The most uniform effect, as we go from 150 to $2,400 \mathrm{msec}$ of viewing time, is a progressive recruitment of item information at all display locations. Taking our results in conjunction with those of Townsend. Taylor. and Brown (1971). we evidently may conclude that there are severe limitations on an individual's capacity for report from a single tixation. even when the role of memory is negligible and when there is no problem of readout from a fading trace in the visual system. Major sources of capacity limitation and many of the salient properties of serial position functions must lie in factors intrinsic to the processes of feature extraction and interpretation.

Laterality. A comparison of data for the left and right visual fields shows somewhat higher reportability of items in the right visual field at all exposure durations, in agreement with the results of Wolford and Hollingsworth (1974b), among others. However, we tind very little difference between the left and right fields with respect to the correct positioning of letters that are reported. The finer analysis of errors reveals no difference between the left and right fields in confusion errors, but a smaller incidence of omission errors on the right. especially at the shorter duration. This latter effect evidently cannot be attributed to habits of reading out material from left to right, since the same difference appears in our data whether the subjects are instructed for left-right or right-left report orders. We cannot be sure that the more rapid and more efficient extraction of item information from the right visual field reflects a laterality effect with respect to the cortical hemispheres, but, on the other hand, we lack any other obvious interpretation.

Central-peripheral gradients. Two distinct effects have been demonstrated with respect to the position of a letter in the visual field relative to the fixation point. First. measures of item information fall off sharply from center to periphery, the correct positioning of reported items somewhat less so. Second, transposition errors tend to move centrally, whether the transposed item displaces another letter or occupies the position of a letter which was not reported from the given display.

Interactions between characters. Cutting across the other factors. large effects of the relative location of items within a display are apparent both in item and in position measures. The serial position functions are uniformly concave upward, with performance best at the end positions. The mutually interfering effects of stimulation from neighboring letters are most strikingly manifest in what we have termed the discontinuity effect. For all response measures, we find performance for the letter in the outermost position of an inner quadrant to be superior to that of the letter in the innermost position of an outer quadrant, even though the quadrants overlap so that these letters are in the same locations with respect to distance from the fixation point. In general, it appears that a letter suffers more interference from other letters than from any of the nonletter mask characters we have used. the difference being larger at increasing distances from the fixation point and the effect being directed predominantly from the periphery towards the center of the field.

Effects of change vs. no change in mask characters. It is well known that perception of letters generally suffers when they are adjacent to any type of mask character in contrast to their perceptibility when adjacent to blank spaces. Further. Estes, Bjork. and Skaar (1974) have shown that this effect of adjacent mask characters is substantially greater if the mask characters change at the time of display onset than if they remain unchanged while the displayed letters appear and disappear, this result being obtained with a detection procedure and very short exposure 
durations. In the present study, the same effect appears much magnified with a report procedure and greatly extended viewing time. Regardless of exposure duration. we have obtained a large advantage for letters which appear adjacent to unchanging mask characters, this effect being especially conspicuous at the left periphery and stronger for item than for position measures.

\section{Bearing of Results on Theoretical Processes}

Scanning processes. A favorite source of explanation of serial position effects in tachistoscopic perception has been the hypothesizing of various serial processes. These hypotheses fall into three categories, which require separate consideration.

(1) Serial. item-by-item processing of letters at input. presumably during the stage of extraction of feature information, is assumed in the hypothesis of Geyer (1971) of a left-right scan at the rate of $8 \mathrm{msec} /$ letter during input processing and the hypothesis of Coltheart (19.72) of a scan at the rate of about $20 \mathrm{msec} / \mathrm{character}$, but directed outside in, that is, beginning at the extremes of a letter display and proceeding inward toward the fovea.

Our study is not designed to bear on these interpretations, and we have only a couple of relevant observations to contribute. First, the fact that processing appears to be more rapid in the right visual field than in the left does not seem especially compatible with the idea of left-right directional processing. The "outside-in" scan was posited to account for the increased perceptibility of letters at the ends of a string and the observation that these positions are selectively affected by forward masking. However, the increased perceptibility at the ends of a string can be accounted for more parsimoniously on other grounds (see below). With regard to the selective effect of forward masking, we would only suggest the possibility that this effect, like that of changing vs. unchanging noise characters at the ends of letter displays. may instead have to do with the distinction between information available only at display onset and information available during the steady-state condition subsequent to onset. The first type of information would have to do with the determination by the processing system of which locations in an array contain letters, the second type with the comparison of particular features with representations in memory and determination of which of possible alternative letters should be reported from each location.

(2) The second type of scanning hypothesis, associated for example with Harcum (1967), includes the assumption that visual information is entered in parallel but then is converted to letter representations by a serial scan of the facing traces of the input in a short-term visual memory system. The right visual field advantage generally observed at short exposure times when, as in our study, displays are presented unilaterally, has been interpreted in terms of this scanning hypothesis (Harcum. 1966). It is assumed that the letters of a display (or their traces) are scanned in a left-right order and that the scanner takes longer to move from a central fixation point to the first letter of a display in the left than to the first letter of one in corresponding positions of the right visual field. This interpretation does not seem well in accord with our finding that, at 150-msec exposure, the proportion correct is lower at Position 1 than at Position 4 in Quadrant 4 (Figure 2). Further, the right visual field advantage is evident even at exposure durations of $600-2,400 \mathrm{msec}$ when exposures are terminated by eye movements (Figure 4). If scanning of fading traces does occur, it evidently plays no major role in generating the characteristic properties of serial position curves, since, as we have seen repeatedly in this study, these properties are strikingly unaffected by very large variations in viewing time.

(3) The third category, represented by the model of Sperling (1967), involves simply the assumption that the output of the visual processing system is converted into letter representations in the auditory-articulatory system by a serial process which proceeds at a rate of the order of $100-200 \mathrm{msec} / \mathrm{item}$. Nothing in our data seems incompatible with this conception. The conspicuously high performance of our subjects in reporting items at the leftmost ends of display strings, especially in the leftmost quadrant, might be taken as suggestive evidence that the process of organizing letter representations for output proceeds in a left-right direction. This tendency must evidently extend to a deeper level than simply habits of reporting from left to right, for requiring our subjects to report from right to left produced only a small shift in the left-right asymmetry of the serial position curves (and a comparable result has been reported by Wolford and Hollingsworth, 1974b). If output is organized in a left-right sequence, then we might expect a tendency for transposition errors to move to the left. This tendency is indeed apparent in our data. In Table 4, for example, the excess of leftward over rightward transpositions in the right quadrants is much larger than the excess of rightward over leftward transpositions on the left. Further, we have noted a conspicuous tendency for letters presented at Positions 2 or 3 of a string to transpose into Position 1 if the letter presented at Position 1 is not reported from the given display.

In general. it has been our strategy not to concentrate on detailed testing of scanning hypotheses of the first two types at present. but rather to attempt to bring out specific sources of properties of serial position curves which can be localized in properties of the display or in interactions among elements of the display and thus perhaps to reduce the set of phenomena which might be taken to call for the 
postulation of high-speed internal scanning processes. We turn now to consideration of the two principal classes of determinants we can identify which do not have to do with scanning processes, namely, interactions among letter inputs and positional uncertainty.

Lateral interference. It has been well known for a long time that, at an observable level, perceptibility of a letter tends to be reduced by the presence of other letters in its immediate neighborhood in a display (see, for example, Woodworth, 1938). In the recent literature, phenomena falling in this category have frequently been referred to under the rubric lateral masking (Estes, 1972; Townsend, Taylor, \& Brown, 1971; Wolford \& Hollingsworth, 1974a), with the implication that an interaction occurs at a level of sensory processing such that stimulus input from one letter reduces the likelihood that feature information can be extracted from other letters in the display, the effect varying inversely with interletter separation and increasing with distance from the fovea. Presumably, an effect at this level would be expected on the basis of the neurologically oriented model presented by Walley and Weiden (1973).

Now we ask what firm evidence can be pointed to in the present results that these phenomena represent a perceptual interaction rather than effects that might be attributable to decision processes or response interference. The data on clarity ratings might perhaps be taken to point in the direction of a perceptual interaction; evidently, letters appear clearer to the subjects when they occur at the ends of a string and thus on one side are adjacent to an unchanged mask character rather than to another letter. A second possibly relevant bit of evidence has to do with the effect of changing vs. nonchanging mask characters flanking a display; these primarily affect the letters in the end positions and do so by increasing the frequency of omission errors, apparently reflecting a degradation of item information. A third type of evidence has to do with the specific relationships between particular flanking mask characters or pre- and postmasks and specific types of letters. In agreement with Wolford and Hollingsworth (1974a), we find differential effects of different mask characters on adjacent letters which have a vertical line segment at the left side as distinguished from letters which do not. However, it should be pointed out that none of these sources of evidence indicate definitely that the lateral interference effects are greater for letters than for mask characters which are adjacent to a target letter, provided that the adjacent characters change at display onset simultaneously with the appearance of the target letter.

Thus, for our experimental conditions, it appears that there is an effect that can reasonably be termed lateral masking, but that the effect is not specific to letters as opposed to other characters and therefore may have nothing to do with interactions at the level of critical features. It should be noted, in this connection, that the interletter spacing in our study was considerably greater ${ }^{2}$ than that used by Wolford and Hollingsworth (1974a), so the possibility remains that a type of inhibitory interaction might occur in their experiment which did not in ours.

The positional uncertainty hypothesis. What can be said about the nature of the lateral interference effects that are not attributable to masking, as the term has been delimited above? Our findings concerning the substantial frequency of transposition and orderinversion errors, even at extended viewing times, suggests a major role for a factor that might be termed positional uncertainty.

It appears that a number of findings on lateral interference can be accounted for parsimoniously on the assumption that the information an observer extracts and enters in immediate memory concerning the location of a displayed letter can be described by an uncertainty gradient around the true location. This function would have properties much like the familiar generalization gradient for conditioned stimuli, but the abscissa in this case would represent distance on a line from the fixation point through the true location of the displayed letter and the ordinate the probability that the observer assigns the letter to a given position in constructing his report of the display. In view of the increasing frequency of positional errors from center to periphery of the visual field and the central drift of transpositions, we would assume that the uncertainty gradient is skewed in the direction of the fixation point, with its variance increasing from center to periphery. These properties would be suggested also by the model proposed by one of the authors (Estes, 1972), which assumes that input channels to feature detectors can be conceived to be distributed over the visual field with the density greater near the fovea and decreasing toward the periphery.

In terms of these ideas, we should expect lateral interference between letters to be manifest whenever their uncertainty gradients overlap. When an observer is attempting to read out a given display location and the uncertainty gradient of the letter displayed there overlaps that of a neighboring letter, it will be difficult for the observer to place the letters in their correct locations. Representations of both letters may be simultaneously activated in the memory system and, in effect, competing for access to the response system. This situation poses a decision problem which is not always resolved correctly, such as in the analogous case of signal detection theory, hence the occurrence of transposition and order-inversion errors.

If, however, the two letters whose uncertainty gradients overlap constitute part or all of a familiar letter sequence, then the uncertainty is resolved in favor of the familiar order. Thus, if the positional 
gradients of adjacent letters $H$ and $T$ overlap, the decision process tends to result in output of $\mathrm{TH}$ rather than of HT. This conception accounts for our finding that the frequency of digrams in English is correlated with the frequency of order-inversion errors involving these digrams in our data but is not correlated with the frequency of report of the letters constituting the digram (regardless of order).

When, under the no-change condition of our study, a letter occurs at one of the end positions of a display, its nearest neighbor on one side is an unchanging mask character. In this case, even though the uncertainty gradients of the end letter and the adjacent mask character overlap, the mask character is readily rejected at the decision level and lateral interference is reduced, thus yielding the concave upward serial position curve. Further, in view of the skewness of the uncertainty functions, the gradient for a neighboring letter on the peripheral side of a target letter will overlap that of the target letter more than would be the case for a neighboring letter on the central side. Consequently, replacing a peripheral neighbor by an unchanging mask character or by a blank space has a greater facilitating effect on reportability of a target letter than replacing a central neighbor (Estes \& Wolford, 1971; Shaw, 1969; Wolford \& Hollingsworth, 1974a). However, replacing the peripheral neighbor of a letter with a mask letter that changes at display onset would not have the same effect according to the positional uncertainty hypothesis, for the changing mask character could not be rejected unequivocally at the decision level as a possible letter. Consequently, if both the end letter of a display and an adjacent mask character which appears simultaneously are far enough from the fixation point so that their uncertainty gradients overlap appreciably, the observer will be unsure as to which of the degraded inputs belongs to the end letter position and thus his error probability will be increased over the cases where the neighbor is an unchanged mask character or a blank space.

A rather similar conception, but one emphasizing discriminability of location, has been suggested by Eriksen and Eriksen (1974) in connection with the interpretation of data from their study and from that of Eriksen and Hoffman (1973) showing that letters which are associated with different classification responses appear to interfere with each other, in the sense of increasing reaction time, more strongly as they are brought closer together within a display, and that this effect appears to be independent of visual similarity of the letters. Similarly, Holmgren (1974) has found that the rate of search for a target letter is slowed significantly if there is another letter in the vicinity of the target which would be expected to lead to a conflicting response. In Holmgren's study and in those of Eriksen and his associates, exposure conditions were such that all of the letters involved could no doubt be identified perfectly. Nonetheless, in terms of our conception, the spatial uncertainty gradients of letters presented close together may overlap, with the consequence that the observer must obtain and process additional information in order to resolve his uncertainty as to which of several possible letters he should assign to the target location, hence the increase in reaction time with decreasing distance between letters which require different responses.

A remark on terminology. In view of the considerations discussed in the preceding paragraphs, we are inclined to suggest very tentatively that it would make for clarity if the term lateral masking were reserved to refer to interference among elements of a display which depends on their physical properties but not on their properties as constituents of an alphabet, that is, not on their makeup in terms of critical features or the like. It appears that masking, so defined, does not extend over intercharacter separation of more than about $0.5^{\circ}$ of visual angle (Eriksen \& Rohrbaugh, 1970). The term lateral interference would encompass the broader class of effects which extend over substantially greater distances in the visual field and which may have their origin primarily in positional uncertainty and response competition.

\section{REFERENCES}

BJork. E. L., \& Estes, W. K. Letter identification in relation to linguistic context and masking conditions. Memory \& Cognition. 1973, 1, 217-223.

BRYDEN, M. P. Accuracy and order of report in tachistoscopic recognition. Canadian Journal of Psychology, 1966, 20, 262-272.

Coltheart, M. Visual information-processing. In P. C. Dodwell (Ed.). New horizons in psychology (Vol. 2). Baltimore, Md: Penguin Books, 1972. Pp. 62-85.

Crovitz, H. F., \& Schiffman, H. R. Visual field and the letter span. Joumal of Experimental Psychology, 1965, 70, 218-223.

Errksen, B. A., \& Eriksen, C. W. Effects of noise letters upon the identification of a target letter in a nonsearch task. Perception \& Psychophysics, 1974, 16, 143-149.

ERIrsen, C. W., \& Collins, J. F. Some temporal characteristics of visual pattern perception. Joumal of Experimental Psychology, $1967,74,476-484$.

Eruxsen, C. W., \& Hofmuar, J. E. The extent of processing of noise elements during selective encoding from visual displays. Perception \& Psychophysics, 1973, 14, 155-160.

Ertksen, C. W., \& Rohrbaugh, J. W. Some factors determining efficiency of selective attention. American Journal of Psychology, 1970, 83, 330-342.

Estes, W. K. Interactions of signal and background variables in visual processing. Perception \& Psychophysics, 1972, 12, 278-286.

Estes, W. K. Redundancy of noise elements and signals in visual detection of letters. Perception \& Psychophysics, 1974, 16. 53-60.

Estes, W. K. Memory, perception, and decision in letter identification. In R. L. Solso (Ed.). Intormation processing and cognition. The Loyola symposium. Hillsdale, N.J: Erlbaum Associates, 1975. 
Estes, W. K., Bjork, E. L., \& SkaAR, E. Detection of single letters and letters in words with changing versus unchanging mask characters. Bulletin of the Psychonomic Society, 1974, 3. 201.203.

Estes, W. K., \& Wolford, G. L. Effects of spaces on report from tachistoscopically presented letter strings. Psychonomic Science, $1971,25,77-80$.

GEYER, J. J. Modeis of perceptual processes in reading. In H. Singer \& R. B. Ruddell (Eds.), Theoretical models and processes of reading. Newark, Del: International Reading Association, 1971.

HARCUM, E. R. Visual hemifield differences as conflicts in direction of reading. Journal of Experimental Psychology, 1966, 72, 479-480.

Harcum, E. R. Parallel functions of serial learning and tachistoscopic pattern perception. Psychological Review, 1967, 74, 51-62.

Hershenson, M. Perception of letter arrays as a function of absolute retinal locus. Journal of Experimental Psychology, 1969. 80, 201-202.

Holmgren, J. E. The effect of a visual indicator on rate of visual search: Evidence for processing control. Perception \& Psychophysics, 1974, 15, 544-550.

Merikle, P. M., \& Coltheart, M. Selective forward masking. Canadian Journal of Psychology, 1972, 26, 296-302.

Merikie, P. M., Coltheart, M., \& Lowe, D. G. On the selective effects of a patterned masking stimulus. Canadian Journal of Psychology, 1971, 25, 264-279.

NeISSER, U. Cognitive psychology. New York: Appleton-CenturyCrofts, 1967.

REDER, S. M. On-line monitoring of eye-position signals in contingent and noncontingent paradigms. Behavior Research Methods \& Instrumentation, 1973, 5, 218-228.

SHAw, P. Processing of tachistoscopic displays with controlled order of characters and spaces. Perception \& Psychophysics, $1969,6,257-266$.

SPERLING, G. Successive approximations to a model for short-term memory. Acta Psychologica, 1967. 27, 285-292.
TAYlor, S. G., \& Brown, D. R. Lateral visual masking: Supraretinal effects when viewing linear arrays with unlimited viewing time. Perception \& Psychophysics, 1972, 12, 97-99.

Townsend, J. T., TAylor, S. G., \& BRown, D. R. Lateral masking for letters with unlimited viewing time. Perception \& Psychophysics, 1971, 10, 375-378.

Underwood, B. J., \& Schulz, R. W. Meaningfulness and verbal behavior. Chicago: Lippincott, 1960.

WALLEY, R. E., \& WEIDEN, T. D. Lateral inhibition and cognitive masking: A neuropsychological theory of attention. Psychological Review, 1973, 80, 284-302.

Winnick, W. A., \& Bruder, G. E. Signal detection approach to the study of retinal locus in tachistoscopic recognition. Journal of Experimental Psychology, 1968, 78, 528-531.

Wolforo, G. L., \& Hollingsworth, S. Lateral masking in visual information processing. Perception \& Psychophysics, 1974, 16, 315-320. (a)

Wolford, G. L., \& Hollingsworth, S. Retinal location and string position as important variables in visual information processing. Perception \& Psychophysics, 1974, 16, 437-442. (b) Woodworti, R. S. Experimental psychology. New York: Holt, 1938.

\section{NOTES}

1. It is to be understood that our conclusions regarding effects of viewing time are limited to conditions in which exposure of a display is terminated by a pattern mask.

2. However, the two studies were similar with respect to the ratio of interletter spaces to letter width. The role of this factor requires further investigation.
(Received for publication May 2, 1975; revision received August 18, 1975.) 